University of Nebraska - Lincoln

DigitalCommons@University of Nebraska - Lincoln

Faculty Publications from the Department of Electrical \& Computer Engineering, Department Electrical and Computer Engineering

2004

\title{
Investigation of Femtosecond Laser-assisted Micromachining of Lithium Niobate
}

\author{
A. Malshe \\ University of Arkansas, Fayetteville \\ D. Deshpande \\ University of Arkansas, Fayetteville \\ E. Stach \\ National Center for Electron Microscopy, Lawrence Berkeley Labs \\ Kamlakar Rajurkar \\ University of Nebraska-Lincoln, krajurkar1@unl.edu \\ Dennis R. Alexander \\ University of Nebraska-Lincoln, dalexander1@unl.edu
}

Follow this and additional works at: https://digitalcommons.unl.edu/electricalengineeringfacpub

Part of the Electrical and Computer Engineering Commons

Malshe, A.; Deshpande, D.; Stach, E.; Rajurkar, Kamlakar; and Alexander, Dennis R., "Investigation of Femtosecond Laser-assisted Micromachining of Lithium Niobate" (2004). Faculty Publications from the Department of Electrical and Computer Engineering. 105.

https://digitalcommons.unl.edu/electricalengineeringfacpub/105

This Article is brought to you for free and open access by the Electrical \& Computer Engineering, Department of at DigitalCommons@University of Nebraska - Lincoln. It has been accepted for inclusion in Faculty Publications from the Department of Electrical and Computer Engineering by an authorized administrator of DigitalCommons@University of Nebraska - Lincoln. 


\title{
Investigation of Femtosecond Laser-assisted Micromachining of Lithium Niobate
}

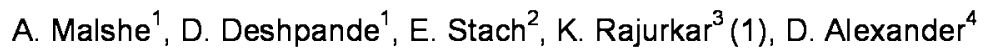 \\ ${ }^{1}$ Department of Mechanical Engineering, University of Arkansas, Fayetteville, USA \\ ${ }^{2}$ National Center for Electron Microscopy, Lawrence Berkeley Labs, Berkeley, USA \\ ${ }^{3}$ Department of Industrial and Management Systems Engineering, University of Nebraska, Lincoln, USA \\ ${ }^{4}$ Department of Electrical Engineering, University of Nebraska, Lincoln, USA
}

\begin{abstract}
Lithium Niobate has a potential for applications in electronics and communication industries due to its unique electro-optical, piezoelectric and nonlinear properties. Femtosecond laser machining offers the best alternative to machine the mechanically fragile and optically delicate lithium niobate crystal. This paper reports a study of the effect of femtosecond laser machining on the surface integrity of lithium niobate. The transmission electron microscopy reveals a $100 \mathrm{~nm}$ thin amorphous region and a void. The chemical analysis shows a loss of lithium and oxygen from the surface and sub-surface. Optical illumination facilitates the selective readout of the written spots of 2 microns size.
\end{abstract}

Keywords: Laser micro machining, single crystal, surface

\section{INTRODUCTION}

Lithium niobate $\left(\mathrm{LiNbO}_{3}\right)$ in single crystal form is one of the most attractive materials in optoelectronics due to its unique electro - optical, piezoelectric and nonlinear properties which merit its use in varied applications such as 'writing' permanent structures on and sub surface such as durable three-dimensional memory, gratings, waveguides and piezoelectric devices [1]. However, $\mathrm{LiNbO}_{3}$ is difficult to machine using conventional methods like grinding, slicing, chemical etching, etc due to its mechanically fragile and optically delicate nature.

Recently, femtosecond lasers have been effectively demonstrated to machine a wide range of materials at micro as well as nano scales for optical and other applications [2-4]. Femtosecond lasers, unlike their micro and nanosecond counterparts, have distinct advantages over conventional machining methods, such as direct writing of structures, higher processing speeds due to effective optical coupling, minimal contamination of processed material due to the ultrashort pulse width allowing athermal processing and spatial resolutions in the sub-micron regime [5-9]. These advantages are especially important while writing spatially and chemically precise structures such as memory bits and waveguides. Femtosecond lasers have been used to machine $1 \mu \mathrm{m}$ holes in silicon, with aspect ratios upto 8 [7]. It was observed that self-focusing of the femtosecond laser pulse might have occurred during the machining, which might have yielded the high aspect ratio holes.

In addition, the realization of small and repeatable features with this method is particularly challenging as $\mathrm{LiNbO}_{3}$ demonstrates non-linear optical characteristics while interacting with the high - energy laser. It is well known that strong correlations exist between laserinduced modifications and resultant optical properties like photon trapping, light scattering and electro-optic switching. Hence, a detailed study of the effects of femtosecond laser machining on optical materials is essential.

However, there is little or no literature in understanding the effect of femtosecond lasers on the physical and chemical end state of crystalline optical materials like $\mathrm{LiNbO}_{3}$, which has a direct influence on their optical properties. A recent study describes the effect of femtosecond laser processing on lithium niobate and analyzes the end state of lithium niobate after processing [10]. Since the intended functions of these structures are to act as optical elements such as optical memory bits or waveguides, the next step in this direction is to analyze the optical response of these machined structures.

Therefore, this paper presents a study of the interactions between a variable energy Ti-Sapphire $(300 \mathrm{fs}, 800 \mathrm{~nm})$ laser and a (110) oriented single crystal $\mathrm{LiNbO}_{3}$ slab and their optical response after machining. Laser induced structures were written on the surface and subsurface using a single femtosecond laser pulse per structure. Localized changes in the refractive index of the material were effected by micro-machining the sample with a high energy laser. The micro-machined structures obtained were then analyzed using physical, chemical and optical spectroscopy techniques [11]. The second section describes the experimental set-up and measurement/ observation details. The experimental results with analysis are presented in the third section. The possible physical mechanism of the interaction between the femtosecond laser pulse and the material is described in the fourth section. The last section summarizes the investigation.

The site-specific sample preparation capabilities of a focused ion beam (FIB) instrument were exploited to create electron transparent regions of surface and subsurface laser processed $\mathrm{LiNbO}_{3}$. A particularly high energy density was used to machine these structures $\left(123.52 \mathrm{~J} / \mathrm{cm}^{2}\right)$, which is much higher than the damage threshold $\left(2.3 \mathrm{~J} / \mathrm{cm}^{2}\right)$ [11]. The authors chose an energy regime where the laser-induced modifications would be clear to all the applied analytical techniques and also would be of clear interest for the desired application.

The processing was carried out in air under normal conditions. The spot size of the beam was controlled by choosing a suitable focusing objective. The structures were written in the form of three-dimensional arrays of damage spots, spaced $5 \mu \mathrm{m}$ and $10 \mu \mathrm{m}$ apart.

\section{EXPERIMENTS}

The Ti:Sapphire femtosecond laser setup used for processing is shown in figure 1. Low energy pulses are generated in a mode-locked Ti:Sapphire oscillator, pumped by a frequency doubled $\mathrm{Nd} \mathrm{YVO}_{4}$ laser. These 
are then amplified by a Ti:Sapphire regenerative amplifier, pumped by a Nd: YLF laser. The high-energy beam then passes through a shutter, which controls the number of pulses reaching the sample. It then passes through the halfwave plate, the polarizer and finally the microscope objective, which focuses the beam onto the sample. The spot size of the incoming beam was adjusted by choosing a suitable focusing objective (numerical aperture 0.4 ). The sample was kept on a micropositioner (Melles Griot Nanomotion II) to facilitate translation of the sample relative to the incoming pulse. A CCD camera was used as an in-situ vision system. The shutter and the micropositioner are controlled by a computer.

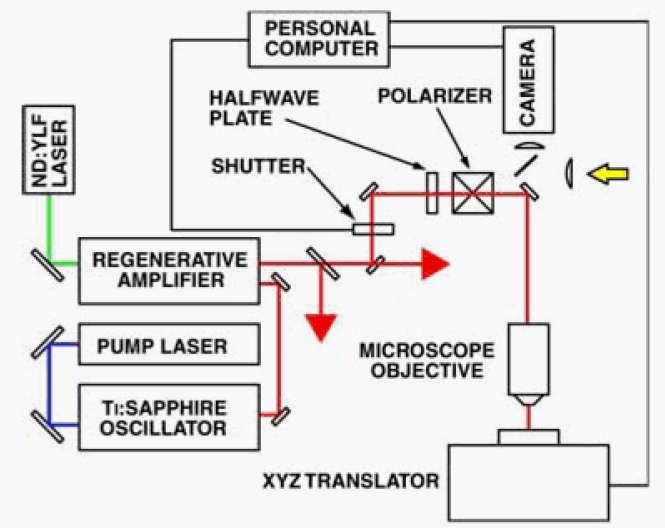

Figure 1: Schematic of laser processing setup.

The wavelength used was $800 \mathrm{~nm}$, with the pulsewidth being 300 femtoseconds. A repetition rate of $67 \mathrm{~Hz}$ was used. Microscope objectives with numerical apertures of 0.3 and 0.4 were used to focus the laser pulse on the sample

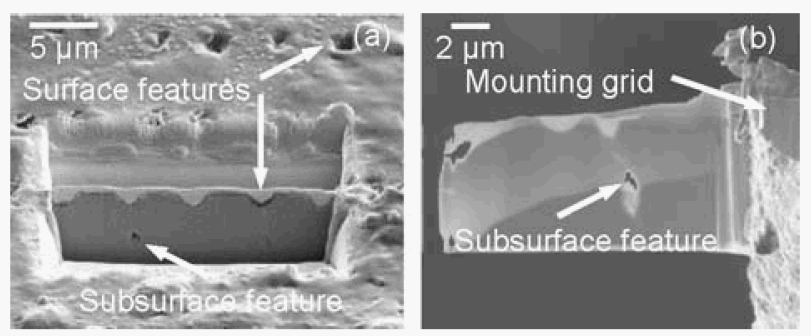

Figure 2: (a) SEM image of sample during FIB membrane preparation; (b) SEM image of a mounted sample.

The microstructural analysis was performed using a combination of a FIB (FEI Strata 235 DualBeam instrument) for sample preparation and a TEM (JEOL 3010) for characterization. The analytical microscopy was performed on a Philips CM200 FEG TEM equipped with the EDS. The TEM samples were prepared in the FIB using the lift-off technique (figures $2 a$ and $2 b$ ) [12]. Figure $2 a$ shows a partially prepared TEM sample. This image was taken with the sample tilted $52^{\circ}$ from the top surface. Individual damage features from both the surface and sub-surface features are observed. Figure $2 b$ shows an SEM image of a different membrane following lift-out and platinum welding to the TEM mounting grid. The difference in contrast in the membrane is a result of different thickness of the sample in these two areas.

\section{ANALYSIS AND DISCUSSION}

A bright field TEM image of one of the surface processed features (the center one of the membrane in figure $2 a$ ) is shown in figure $3 a$. The carbon and platinum layers seen here are a part of the FIB sample preparation process, and are not a part of the original sample. Below these layers, the semicircular contour of the locally ablated sample is seen - it has a diameter of about $2 \mu \mathrm{m}$, which relates to the expected spot size of the laser pulse.

Immediately below this contour is an approx. $100 \mathrm{~nm}$ thick layer exhibiting a flat gray contrast: local electron diffraction in the TEM (from the area indicated as $b$ and shown in figure $3 b$ ) clearly indicates that this layer is amorphous. Adjoining this layer is a second region of about $1 \mu \mathrm{m}$ thickness containing highly defective crystalline material, as shown by the electron diffraction pattern taken at $c$ (figure $3 c$ ). Small probe selected area diffraction showed no evidence of crystalline phases other than $\mathrm{LiNbO}_{3}$ in the laser processed region. Although other phases are present in the $\mathrm{Li}_{2} \mathrm{O}-\mathrm{Nb}_{2} \mathrm{O}_{5}$ phase diagram [13], these represent phases formed under thermodynamically stable conditions and are probably not relevant in the predication of any phase changes that might occur during the highly metastable laser processing.

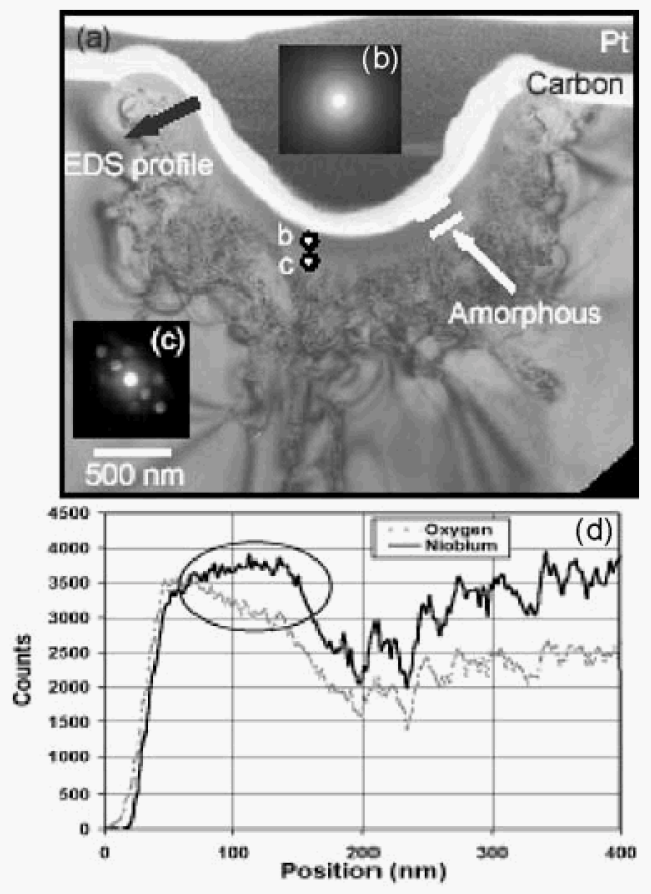

Figure 3: (a) Bright field TEM micrograph of a surface feature from the sample shown as figure $2 a$; (b \& c) Transmission electron diffraction patterns from the areas indicated in figure $3 a$; (d) EDS profile taken from the sample region indicated in figure $3 a$. The circle denotes the area where oxygen deficiency is observed.

The EDS profiles of oxygen and niobium (figure $3 d$ ) beyond approx. $200 \mathrm{~nm}$ can be considered to represent untreated $\mathrm{LiNbO}_{3}$. It is observed that the first $100 \mathrm{~nm}$ of the profile (which is the same size as the amorphous layer) shows evidence of deficiency in oxygen counts, i.e. the amorphous region has a gradient in oxygen content from sub-stoichiometric towards stoichiometric at the start of the crystalline region. These observations are in broad agreement with earlier work [14], where it was predicted that picosecond ablation of $\mathrm{LiNbO}_{3}$ might remove oxygen and lithium from the crystal by analogy to halogen emission from alkali halides.

Figure 4a presents a bright field TEM micrograph showing both surface and sub-surface damage features from a different sample. The surface feature in the upper left corner of the micrograph appears very similar to that of figure 2a: a $2 \mu \mathrm{m}$ diameter crater, surrounded by a 100 $\mathrm{nm}$ thick amorphous region and a heavily defective region of about $0.5-1 \mu \mathrm{m}$ thickness containing lattice defects 
such as dislocations and twins. However, the sub-surface feature has a strikingly different appearance. The focusing of the laser below the surface has resulted in an ellipsoid amorphous core structure about $1 \mu \mathrm{m}$ thick along its minor axis and $3 \mu \mathrm{m}$ in the laser beam direction, indicative of self-guided optical machining [9]. This amorphous region is significantly larger than that associated with the surface damage features and extends for several microns above and below (not shown fully in this imaging condition) the core. Similar observations of amorphization have been reported in quartz, where nano- and micro-joule laser pulses have created amorphous regions sub-surface [15] and in silicon, where amorphization was observed for lower fluence $\left(<0.2 \mathrm{~J} / \mathrm{cm}^{2}\right)$ but not for higher fluence [16]. Additionally, in the work of Gorelik et al. [15] it was shown that the processing left a strain in the quartz, resulting in an increase in the refractive index around the irradiated region. A void is observed at the top of the subsurface structure.

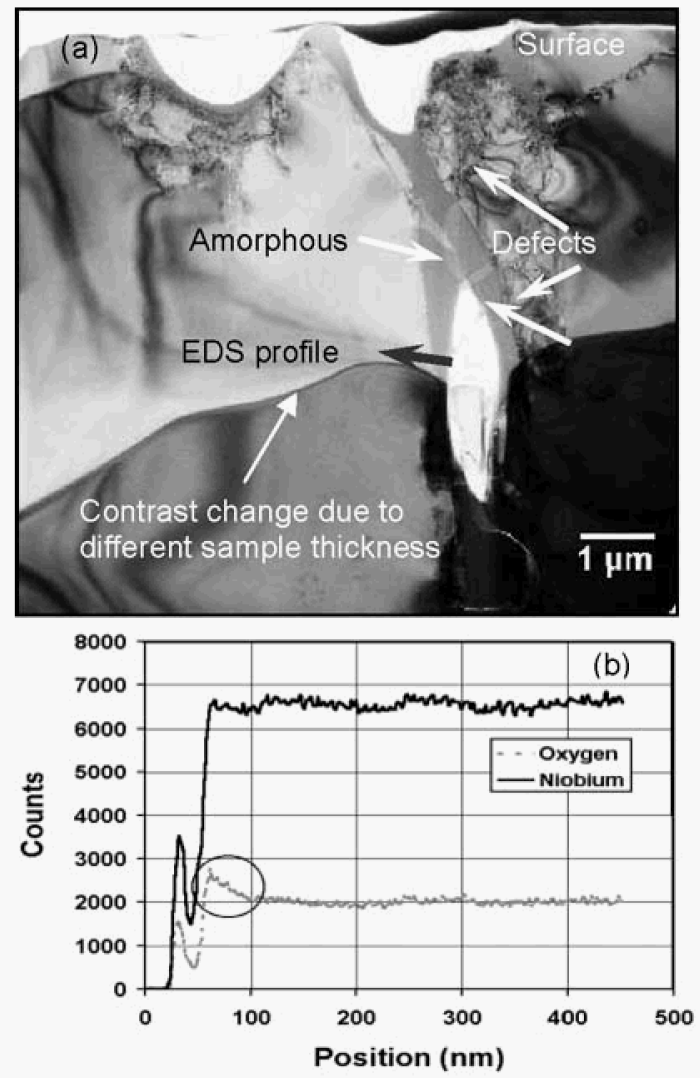

Figure 4: (a) Bright field TEM micrograph of the sample shown as figure $2 b$ with both surface and sub-surface features visible; (b) EDS profile taken from the sample region indicated in figure $4 \mathrm{a}$. The circle denotes the area where oxygen enrichment is observed.

An EDS profile taken across the structure (figure $4 \mathrm{~b}$ ) indicates that the amorphous material near the void has a higher concentration of oxygen than is observed in bulk $\mathrm{LiNbO}_{3}$ - the opposite of the surface feature profiled in figure 3 . This indicates that oxygen has been liberated from the laser-processed area, as was observed for the surface processing. However, in this case, since the liberated oxygen cannot escape to the atmosphere, it instead gets incorporated into the amorphized material at a super-stoichiometric concentration.

After the laser processing, the damage structures were illuminated using a $635 \mathrm{~nm}$ wavelength diode laser [11]. The $\mathrm{LiNbO}_{3}$ slab was placed on an $X-Y$ motion stage (developed in-house), and the output of the diode laser was focused on the damage structures on the slab. Figure
5 shows the illumination of laser created damage structures on the surface of $\mathrm{LiNbO}_{3}$, viewed under normal microscope and laser diode illuminations. The structures were written using five femtosecond laser pulses per structure, and variable power per column of structures. The laser spot size was approximately $3 \mu \mathrm{m}$ in diameter. The power was varied from $34.54 \mathrm{~J} / \mathrm{cm}^{2}$ for the first column of structures, and was reduced in steps to 1.77 $\mathrm{J} / \mathrm{cm}^{2}$ for the $11^{\text {th }}$ column. Accordingly, the diameters of the structures varied from about $6 \mu \mathrm{m}$ to about $0.5 \mu \mathrm{m}$. The $7^{\text {th }}$ and the $8^{\text {th }}$ columns have damage structures with structures equivalent to the laser spot size.

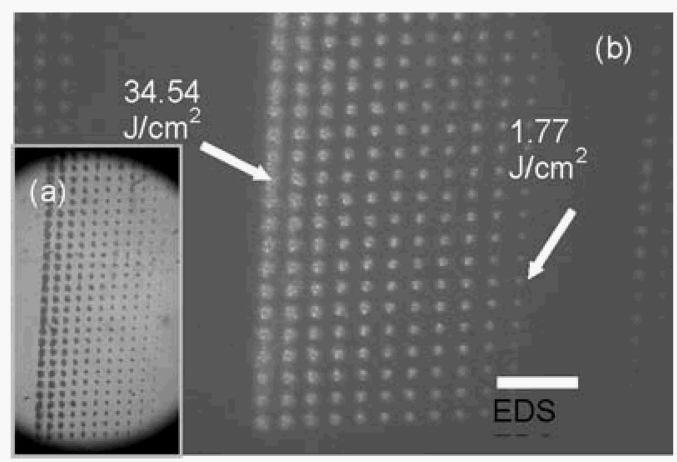

Figure 5: Optical microscope images of an array of damage spots: (a) Illumination provided by microscope light; (b) Image of the same damage spots when illuminated by the $635 \mathrm{~nm}$ wavelength diode laser

As seen from figure $5 \mathrm{a}$, the $6 \mu \mathrm{m}$ structures $\left(1^{\text {st }}\right.$ column) appear to spread extensively around the focal point of the laser. This is due to the spatial spread of the amorphous and especially the damaged regions around the core structure, similar to those seen in the TEM image in figure $3 a$. The size of these regions gradually reduces as one approach the damage threshold of $1 \mathrm{~J} / \mathrm{cm}^{2}$ for $\mathrm{LiNbO}_{3}$ for the given set of parameters. This image was observed under the normal illumination of an optical microscope. This illumination was then switched off and the diode laser was switched on. The illumination as seen in figure $5 b$ is coming from the top of the image. The intensity of illumination of the damage structures depends on the spread of the damaged region around the core structure. The structures written at $34.54 \mathrm{~J} / \mathrm{cm}^{2}\left(1^{\text {st }}\right.$ column $)$ have extensive damage around the core structure, so that adjoining structures appear to have merged. In contrast. the structures written at $1.77 \mathrm{~J} / \mathrm{cm}^{2}\left(11^{\text {th }}\right.$ column $)$ appear tiny and are barely visible. As mentioned above, the structures in the $7^{\text {th }}$ and $8^{\text {th }}$ columns (written at energy densities of $7.97 \mathrm{~J} / \mathrm{cm}^{2}$ and $6.19 \mathrm{~J} / \mathrm{cm}^{2}$ respectively) have sufficient intensity of the reflected laser light, and have physically distinct structures. These energies (approximately 6-8 times the threshold) would produce the optimum size of the damage structures.

\section{MODEL OF FEMTOSECOND ABLATION}

In addition to allowing the detailed characterization of the end state of the material, the cross-sectional TEM observations have given considerable insight into the physics occurring during the interaction between the material and the femtosecond laser pulse. The prevailing observation is that of competing processes - ablation and partial re-deposition, thermal shock and extreme quenching, as well as effects associated with shock wave propagation. Under same energy density conditions in surface and sub-surface processing, ablation-induced material removal is clearly observed as has been reported for quart [15]. However, unlike prior observations in silicon and quart $[15,16]$, the processing of $\mathrm{LiNbO}_{3}$ 
results in formation of an amorphous layer surrounding the laser-ablation region at this high-energy flux.

There are two processes that may lead to this observed amorphization: (1) transient two-photon absorption, followed by heating and quenching, and/or (2) transient absorption followed by ablation and the generation of reaction shock wave. In addition, there will be a redeposition of ablated material: complete in the case of sub-surface exposure, or partial in the case of surface exposure. Further, the observed microstructures suggest that both these mechanisms are competing. This competition results in a shallower amorphous region for the surface features, whereas with the sub-surface processing an ablation and shock wave assisted cavitation and amorphization occur. The quenching and/or shockwave further yield a highly defective region surrounding the amorphous region. The strain caused by this highly defective region extends for about a micron around the sub-surface feature. Since this strain will locally modify the optical properties of the material, the observed 'bit' size (if the feature is considered as a data bit used in memory writing) will be that of this larger damaged region.

Additionally, for the ultrashort laser ablation used here, we see fragmentation of $\mathrm{LiNbO}_{3}$ and the release of oxygen, as has been predicted previously [14]. In the case of surface writing, this oxygen escapes to the atmosphere, whereas the oxygen released in the sub-surface region is incorporated with the redeposited material, and is observed to segregate near the surface of the amorphous region. The formation of the cavity/void during the subsurface processing was surprising. It can be speculated that the cavitation is a result of densification or expansion, causing the ejection of a proportional amount of material, perhaps assisted by a shock wave that propagates through the sample following the pulse. It is important to note that this cavity is not a direct result of the laser flux, but is a secondary occurrence.

\section{SUMMARY}

The micro- and nanoscale surface and sub-surface defects in $\mathrm{LiNbO}_{3}$ formed during femtosecond laser processing have been characterized using ion and electron microscopies. The ablated region is surrounded by amorphized material as well as a region containing highly defective crystal. The sub-surface structure is completely amorphous with a void at the top of the structure, and an amorphous channel that reaches the surface as a result of the explosive nature of the materiallaser interaction. Illumination studies of the machined structures reveal that the 'optical' size of these structures depends on the spatial spread of the damaged crystal region around the core structure. Such understanding is important in achieving better spatial, structural, chemical and hence optical resolution for scaling up in 3-D optical memory and related light-guiding applications.

\section{ACKNOWLEDGEMENTS}

The authors acknowledge the help of Dr. Eric Stach of the Lawrence Berkeley laboratories for the TEM sample preparation. The authors are thankful to Dr. Z. Yu for his assistance in the preparation of the manuscript. This work is supported by the National Science Foundation (NSF) EPSCoR grant \# 9977830.

\section{REFERENCES}

[1] McPherson M., Ostrovskii I., Breazeale M., 2002, Observation of Acoustical Memory in $\mathrm{LiNbO}_{3}$,
Physical Review Letters, 89/11/2002, 115506-1115506-3.

[2] Davis K., Miura K., Sugimoto N., Hirao K., 1996 Writing waveguides in glass with a femtosecond laser, Optics Letters 21/21/1996, 1729-1731.

[3] Ozkan A., Malshe A., Railkar T., Brown W., 1999 , Femtosecond laser-induced periodic structure writing on diamond crystals and microclusters, Applied Physics Letters 75/23/1999, 3716-3718.

[4] Adhi K., Owings R., Railkar T., Brown W. Malshe A., 2003, Femtosecond ultraviolet $(248 \mathrm{~nm})$ excimer laser processing of Teflon (PTFE), Applied Surface Science 218/1-4/2003, 17-23.

[5] Meijer J., Du K., Gillner A., Hoffmann D., Kovalenko V., Masuzawa T., Ostendorf A., Poprawe R., Schulz W.,2002, Laser Machining by Short and Ultrashort Pulses, State of the Art and New Opportunities in the Age of the Photons, Annals of the CIRP 51/2, 531550.

[6] Krüger J., Kautek W, 1999, The Femtosecond Pulse Laser: A New Tool for Micromachining, Laser Physics, 9/1/2000, 30-40.

[7] Alexander D., Mihulka B., Doerr D., 2002 , Femtosecond laser drilling of high aspect ratio $1 \mu \mathrm{m}$ holes in silicon, Proceedings of SPIE, 4760/2002, 383-393.

[8] Tonshoft H. K., et al, 2000, New Applications of Femtosecond Lasers in Micromachining, Proceedings of the 1st Euspen Conference on Fabrication and Metrology in Nanotechnology, Denmark, 10-17

[9] Alting L., Kimura F., Hansen M. N., Bissacco G., 2003, MicroEngineering, Annals of the CIRP, 52/2, 635-657.

[10] Stach E., Radmilovic V., Deshpande D., Malshe A., Alexander D., Doerr D., 2003, Nanoscale surface and subsurface defects induced in lithium niobate by a femtosecond laser, Applied Physics Letters, $83 / 21 / 2003,4420-4422$.

[11] Deshpande D., 2003, Investigation of enabling technologies for Optical Microscope-on-a-Chip, MS thesis, University of Arkansas, Fayetteville, USA

[12] Prenitzer B., Giannuzzi L., Newman K., Brown S., Irwin R., Shofner T., Stevie F., 1998, Transmission Electron Microscope Preparation of $\mathrm{Zn}$ Powders Using the Focused Ion Beam Lift-Out Technque, Metallurgical and Materials Transactions $A$, 29A1998, 2399-2406

[13] Prokhrov A., Kuz'minov $Y_{\text {., 1990, Physics and }}$ Chemistry of Crystalline Lithium Niobate, Adam Hilger series on Optics and Optoelectronics.

[14] Bunton P., Binkley M., Asbury G., 1997, Laser ablation from Lithium niobate, Applied Physics $A$, 65/1997, 411-417.

[15] Gorelik T., Will M., Nolte S., Tuennermann A., Glatzel U., 2003, Transmission electron microscopy studies of femtosecond laser induced modifications in quartz, Applied Physics A, 76, 309-311.

[16] Bonse J., Baudach S., Krüger J., Kautek W., Lenzner M., 2002, Femtosecond laser ablation of silicon-modification thresholds and morphology, Applied Physics A, 74, $19-25$. 